\title{
Mechanisms of phase separation and aggregation in colloid-polymer mixtures
}

\author{
V. J. Anderson, E. H. A. de Hoog, and H. N. W. Lekkerkerker* \\ Van 't Hoff Laboratory for Physical and Colloid Chemistry, Debye Institute, Utrecht University, Padualaan 8, 3584 CH Utrecht, \\ The Netherlands
}

(Received 26 June 2001; published 18 December 2001)

\begin{abstract}
The final structure of a colloidal system is greatly influenced by the mechanisms by which phase separation and aggregation occur. The drive to phase separate can be altered in colloid-polymer mixtures (which phase separate due to the depletion interaction) by varying the polymer concentration. Here, we use small angle light scattering to follow the phase separation in such mixtures and analyze the results within a framework indicated by previous results from microscopy investigations. The mechanisms of diffusion-limited cluster aggregation, reaction-limited cluster aggregation, and nucleation and growth are found to provide good descriptions of the phase separation regimes. The growth rate in the nucleation and growth regime is shown to be dependent on the polymer concentration.
\end{abstract}

DOI: 10.1103/PhysRevE.65.011403

\section{INTRODUCTION}

Depletion-induced aggregation in suspensions of colloidal hard spheres has been the subject of many investigations recently, attention ranging from the fundamental interest in colloids as a simple models of atomic systems, the field of biology, and the understanding of protein crystallization, to the practicalities of industrial applications. The final structure of a colloidal system is very sensitive to the characteristics of the components (polydispersity, for instance) and the interactions between particles as these affect the kinetics of phase separation, making it difficult to predict whether or not a system will crystallize, for example. In colloid-polymer suspensions the interactions can be finely tuned, allowing the different mechanisms to be explored.

It is now generally accepted that phase separation in mixtures of colloids and nonadsorbing polymers is induced by the depletion interaction, a concept introduced by Asakura and Oosawa [1] in 1954. In 1976 this idea was put forward and expanded independently by Vrij [2]. Briefly, the depletion interaction can be thought of in terms of there being a volume surrounding each sphere into which the center of a polymer chain cannot penetrate, thus these volumes are depleted of polymer. If the depletion volumes of two spheres overlap there will be an imbalance in the osmotic pressure due to the depletion of the polymer between the two spheres. The range and strength of the interaction depends on the polymer volume fraction and the size ratio, defined as the ratio of the polymer radius of gyration and the colloid radius. The depletion interaction has been studied theoretically in great detail and the phase diagrams for various colloidpolymer ratios have been predicted (see Verhaegh and Lekkerkerker [3], and references therein). The experimental verification of these phase diagrams has led to some intriguing findings, glassy states, gellike states, and crystalline structures ranging from those that could be termed macroscopic to those that have many defects [4-6]. This variety of outcomes, indicates that the mechanisms by which the homog-

\footnotetext{
*Corresponding author. Email address: H.N.W. Lekkerkerker@
} chem.uu.nl
PACS number(s): 82.70.Dd, 61.43.Hv, 64.60.Qb

enous systems phase separate must be of great importance.

Here, we examine several mechanisms of phase separation, all studied here at low colloid volume fraction. Various models have been proposed to describe phase separation and aggregation phenomena (see, for example, the review by Poon and Haw [7]) and previous work using confocal microscopy has indicated that different models are appropriate for different regions of the phase diagram. Here, we study the same system using small angle light scattering to confirm the various regimes and analyze them in more detail.

The system used here is identical to that used by de Hoog et al. [8]. It consists of samples of poly (methylmethacrylate) (PMMA) spheres, shown in Fig. 1, (at a constant volume fraction of 0.022 ) in a solvent that almost matches both the density and the refractive index. This close matching allows the phase separation process to proceed significantly before the effects of gravity are seen and means that the system can be studied using microscopy and light scattering. Polymer (polystyrene) is added to induce phase separation via the depletion interaction. The ratio of the polymer radius of gyration and the colloid radius is 0.076 . In the previous work by de Hoog et al. [8], confocal microscopy was used to observe the phase separation, at different polymer concentrations $c_{p}$ as it proceeded. Image analysis, which took into

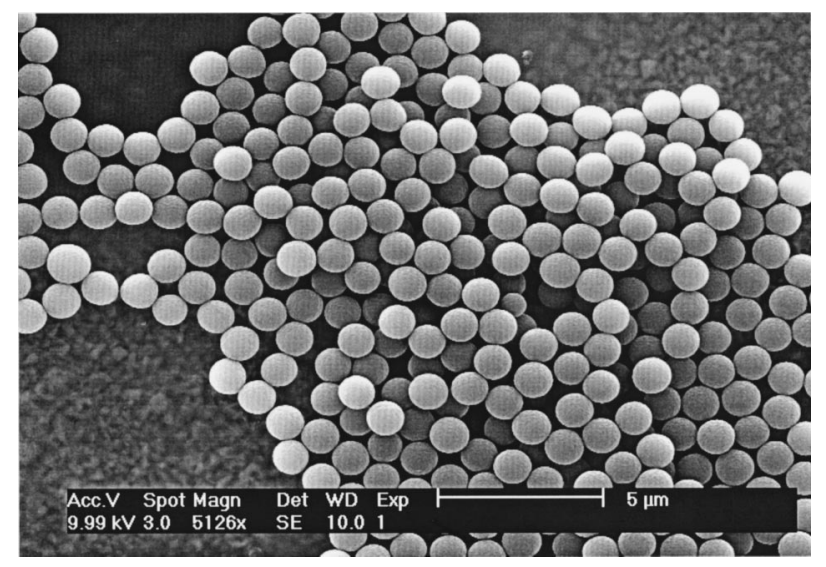

FIG. 1. Scanning electron microscopy image of the NBDPMMA particles used throughout this work. The particles have a diameter of $1.2 \mu \mathrm{m}$. 
account the number of particles per cluster, number of clusters, and the shape of the clusters, has indicated that there are four main regimes: regime $A, c_{p}=0-1.28 \mathrm{mg} / \mathrm{ml}$-no phase separation but enhanced sedimentation; regime $B, c_{p}$ $=1.58-1.72 \mathrm{mg} / \mathrm{ml}$-phase separation via nucleationlike mechanism; regime $C, c_{p}=1.75-2.28 \mathrm{mg} / \mathrm{ml}$ - phase separation via aggregation producing compact clusters; regime $D$, $c_{p}=3.31-9.04 \mathrm{mg} / \mathrm{ml}$ - phase separation via aggregation elongated clusters.

Typical images of samples in regimes $B, C$, and $D$ undergoing phase separation are shown in Fig. 2, the different structures produced by the various mechanisms can easily be seen. It should be noted that the polymer concentrations for these regimes are for this specific system (detailed further in the experimental section), altering the polymer radius of gyration or the solvent mixture, for example, would be very likely to affect the interactions, equilibrium phases, and dynamics of the system. The technique of confocal microscopy used to determine these regimes has the disadvantage in that only two-dimensional images of the sample, effectively slices through the sample, could be examined. (Note that, while it is true that confocal microscopy is a powerful tool for three-dimensional imaging, this was not possible at the speeds of aggregation found in these samples.) The light scattering experiments carried out here investigate the same regimes but have the advantage of examining larger volumes in the sample, thus giving better statistics, and can also be compared with other work on aggregation. Experiments are done at constant colloid concentration ( $2.2 \%$ by volume) and the amount of polymer is varied; increasing the polymer concentration brings the suspensions deeper into the unstable region, thus increasing the drive to aggregate.

It is convenient here to review the models of phase separation that were found in the various regimes. Models of cluster aggregation have been examined theoretically, experimentally, and by simulation [7,9-15]. These identify two important mechanisms of cluster aggregation and describe the characteristic evolution of the structures.

In regime $D$, above, a diffusion-limited cluster aggregation (DLCA) mechanism was found. The model of DLCA assumes that the colloids stick permanently as soon as they touch and thus the mechanism is limited by the time taken for particles to diffuse through the suspension and meet. This results in open structures, since the colloids cannot penetrate deep into a cluster before encountering another particle, and a monodisperse cluster size distribution as the smallest clusters move fastest, have more encounters, and thus are "used up" as reaction proceeds. This is also the regime for which Schmoluchowski kinetics for rapid aggregation should be valid and indeed the samples in regime $D$ showed kinetics in good agreement with the Schmoluchowski predictions.

Deviations from Schmoluchowski kinetics were found in regime $C$, where clusters also appeared to be more compact. These observations led to regime $C$ being classed as showing a reaction-limited cluster aggregation (RLCA) mechanism. The model of RLCA assumes that the particles do not necessarily stick on first contact, thus the aggregation process is limited by the time taken for a "reaction" (or a "sticking encounter") to occur. Usually a "reaction-limited" process is associated with an energy barrier that has to be overcome. In this system there is no barrier, but at low polymer concentrations the drive to phase separate is not strong and there is a significant possibility that particles can move apart, that is, the aggregation can be seen as reversible. This means that within the time for other particles to diffuse to a growing cluster, previous particles may be able to move around the cluster, or indeed break away. Although this picture is not completely analogous to a reaction with a barrier to overcome, it is similar in that particles can move around the cluster and the diffusion of particles towards the cluster is not the limiting step. This process results in more compact structures than the DLCA case as the particles can move further into the cluster before a "reaction" occurs. The size distribution is also more polydisperse than the DLCA case as the smaller particles are not necessarily "used up" first.

On decreasing the polymer concentration further it was found that clusters only appeared after a certain time and the clusters appeared relatively large. This was thus classed as a nucleation and growth regime. In this regime $(B)$ it is expected that the clusters have to be a certain size before they are stable and can grow, this gives rise to an induction period before any growth is seen (see the paper by Feher and Kam [16] for a discussion on nucleation and growth of protein crystals). Nucleation and growth is a common phenomenon in phase separating systems and involves competition between the gain in free energy due to the creation of a nucleus of the new phase and the loss of free energy due to the surface tension of this nucleus. Of course, a cluster with only a few particles does not have the well-defined surface that one normally envisages in a nucleation and growth situation, thus ascribing a surface tension to such a cluster may not be as straightforward, however, the general principles should be similar. The competition between surface and volume terms means that there is a critical cluster size, clusters larger than this will grow, those smaller will dissolve. The time taken for a cluster of this critical size to be formed by chance will depend on the size of the cluster, the larger the critical size the longer the time before a significant number of critical sized clusters will have appeared. The free energy curve as a function of cluster size has a maximum at the critical cluster size, thus the gain and loss of particles does not change the free energy a great deal and the initial growth is slow. As the nuclei become bigger the growth rate increases. Thus, nucleation and growth is characterized by an initial period, where little happens as critical nuclei are appearing and slowly growing, after this delay time the clusters start to grow rapidly.

This paper is organized as follows, we first describe the system and the light scattering equipment used to study it. We then go on to show that the light scattering data agrees with the predictions for DLCA and RLCA aggregation mechanisms in regimes $D$ and $C$, respectively, confirming the results of de Hoog et al. We show measurements of samples in regime $B$ that indicate that nucleation occurs quickly so an induction time is only found for very low polymer concentrations. The growth of the clusters is found to be limited by the probability a particle will remain with the cluster and can be related to the polymer concentration. 


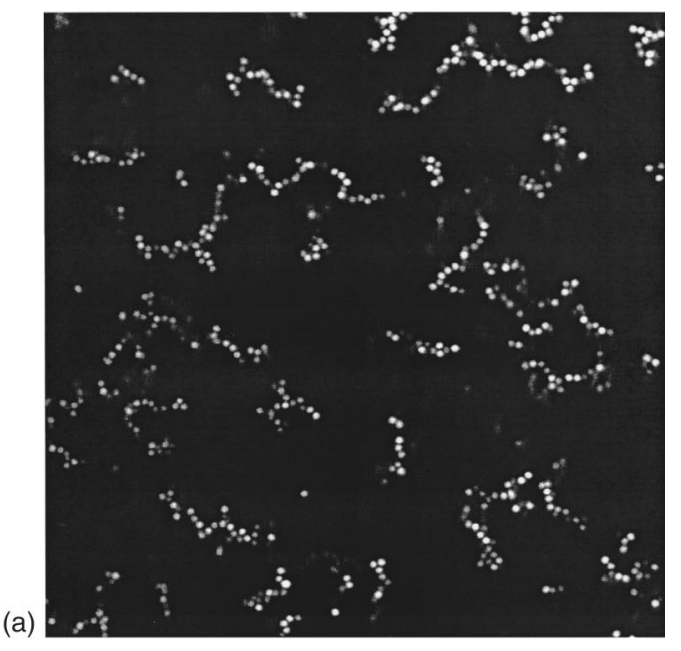

(b)
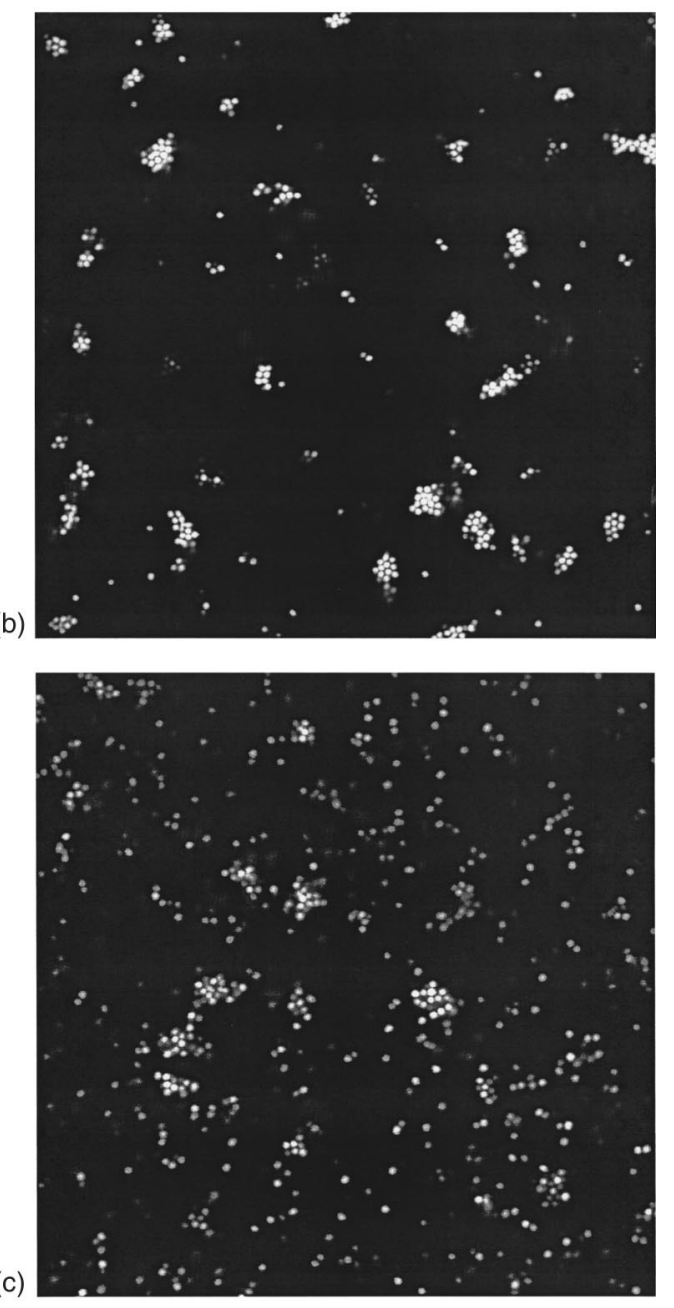

FIG. 2. Typical confocal microscopy images of samples undergoing phase separation. Images show regions that are $100 \mu \mathrm{m}$ by $100 \mu \mathrm{m}$. (a) A sample from Regime $D,\left(c_{p}=\right.$ of $\left.8.07 \mathrm{mg} / \mathrm{ml}\right)$ undergoing aggregation, (image taken at $t=540 \mathrm{~s}$ ). The elongated, stringy clusters typical of DLCA are easily seen. (b) Sample from Regime $C,\left(c_{p}=2.10 \mathrm{mg} / \mathrm{ml}\right)$ at $t=300 \mathrm{~s}$. The clusters are much more compact than those in Regime $D$ and the intercluster distance is larger. (c) Sample from Regime $B,\left(c_{p}=1.72 \mathrm{mg} / \mathrm{ml}\right)$ at $t=1110 \mathrm{~s}$. Even after such a long time only a few nuclei have appeared, these are quite large and are growing in a sea of single particles.

\section{EXPERIMENT}

The system uses PMMA particles prepared by dispersion polymerization at $80^{\circ} \mathrm{C}$ following the method of Antl [17]. The particles were fluorescently labeled with the dye NBD (7-nitrobenzo-2-oxa-1,3-diazol) bonded to a coupling agent (4-methyl aminoethylmethacrylate-7-nitrobenzo-2-oxa-1,3diazol) that was incorporated during the polymerization reaction. A layer of $10 \mathrm{~nm}$ of PHS [poly (12-hydroxystearic acid)], a graft polymer consisting of a backbone of methacrylate and glycidyl methacrylate monomers in a weight ratio of 10:1 and tails of poly (12-hydroxystearic acid), stabilized the particles. The particle synthesis is described elsewhere in more detail [18]. The particles used are shown in Fig. 1. Using confocal scanning laser microscopy and scanning electron microscopy the radius was found to be 600 and 580 $\mathrm{nm}$, respectively, with a polydispersity of $3 \%$. The polymer used is polystyrene of molecular weight $2000 \mathrm{~kg} / \mathrm{mol}$ $\left(M_{w} / M_{n}=1.11\right)$ and its density is $1.11 \mathrm{~g} / \mathrm{ml}$. The radius of gyration of the polymer was found (by viscosity measurements) to be $46 \mathrm{~nm}$ implying an overlap concentration of $8.15 \mathrm{mg} / \mathrm{ml}$. The solvent used was a mixture of tetralin, cisdecalin, and carbon tetrachloride in volume ratios of $36.0 \%$, $31.5 \%$, and $32.5 \%$, respectively. The density of this solvent mixture is $1.148 \mathrm{~g} / \mathrm{ml}$ and the index of refraction is 1.50 . The density of the PMMA particles (determined by sedimentation) is $1.25 \mathrm{~g} / \mathrm{ml}$, thus the density difference is of the order of $0.1 \mathrm{~g} / \mathrm{ml}$. The particles are stable in this solvent mixture for at least several months.

The light scattering apparatus is similar to the apparatus described by Schatzel and Ackerson [19]. Briefly, it consists of a $5 \mathrm{~mW}$ randomly polarized helium-neon laser at a wavelength of $632.8 \mathrm{~nm}$, the beam of which is spatially filtered and expanded using a microscope objective, $25 \mu \mathrm{m}$ pinhole and an $80 \mathrm{~mm}$ focal length output double (Oriel, Stratford, $\mathrm{CT}$ ). The beam passes through the samples and comes to a focus on a distant projection screen. A $5 \mathrm{~mm}$ pinhole limits the main beam near its first diffraction minimum just before the sample. The unscattered beam passes through a hole in the projection screen and the intensity is measured using a photodiode. Variations in the laser beam can be compensated for since a beam splitter between the laser and the spatial filter allows a second photodiode to monitor the main beam. The scattering pattern is recorded by a CCD camera (Electrim, Princeton, New Jersey) with a resolution of $165 \times 192$ pixels with an exposure time in the range 100-400 ms. The range of accessible scattering wave vectors was 100-1800 $\mathrm{mm}^{-1}$. (Note that we define the wave vector such that length scales $r$ and corresponding wave vectors $q$ have the relationship $q=2 \pi / r$.)

Cuvettes with path lengths of $0.1 \mathrm{~mm}$ were used, samples were homogenized by shaking before each experiment. Images were corrected for dark current and stray light by subtracting the first image of time series. Measurements of the intensity of the unscattered beam allowed us to correct for multiple scattering.

\section{RESULTS AND DISCUSSION}

\section{A. Regimes $C$ and $D$}

The small angle light scattering patterns of samples from regimes $C$ and $D$ both show peaks in the intensity appearing 


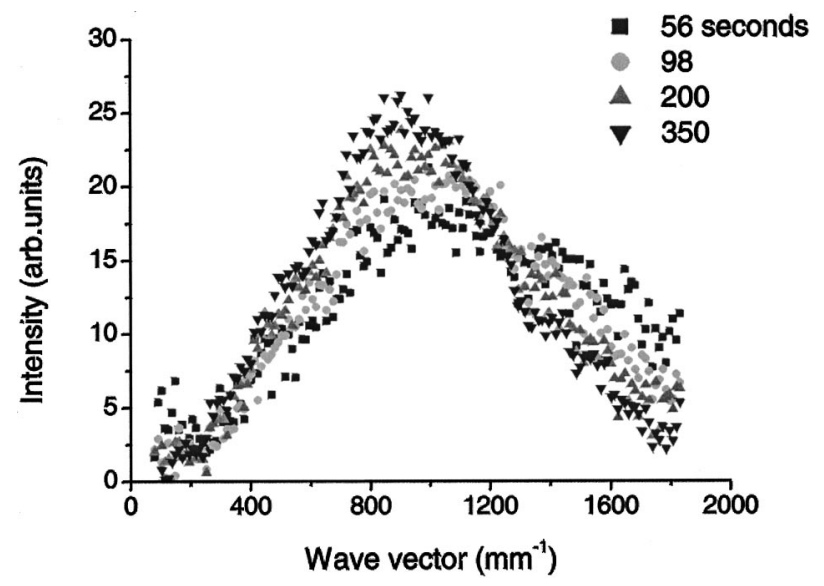

FIG. 3. Typical scattering pattern from a sample in regime $D$ $\left(c_{p}=5.58 \mathrm{mg} / \mathrm{ml}\right)$, showing the evolution of a peak with time. The peak is seen immediately and increases in intensity while moving to smaller wave vectors, as would be expected for a sample undergoing diffusion-limited aggregation.

immediately. This indicates that phase separation occurs immediately. A typical example from regime $D$ is shown in Fig. 3 . This is in agreement with the predictions, in regimes $C$ and $D$ we expect there to be no barrier to phase separation and it will proceed immediately.

The samples show the peak moving to lower wave vectors and increasing in intensity. The peak from the sample in regime $D$ is very symmetrical (see Fig. 4) and is fitted well by a Gaussian. When the curves are scaled by the peak intensity and position (not shown), allowing us to compare different times, the peak stays quite narrow. This implies the cluster size distribution is also symmetrical and that the distribution remains so even at longer times. This is in agreement with the DLCA model described above. In contrast the sample from regime $C$ has a much less symmetric peak, (see Fig. 5) with a long tail at large wave vectors, implying that smaller clusters remain even when other clusters have grown larger. On examining the scaled data, we see that the width of the distribution increases as time goes on, this indicates

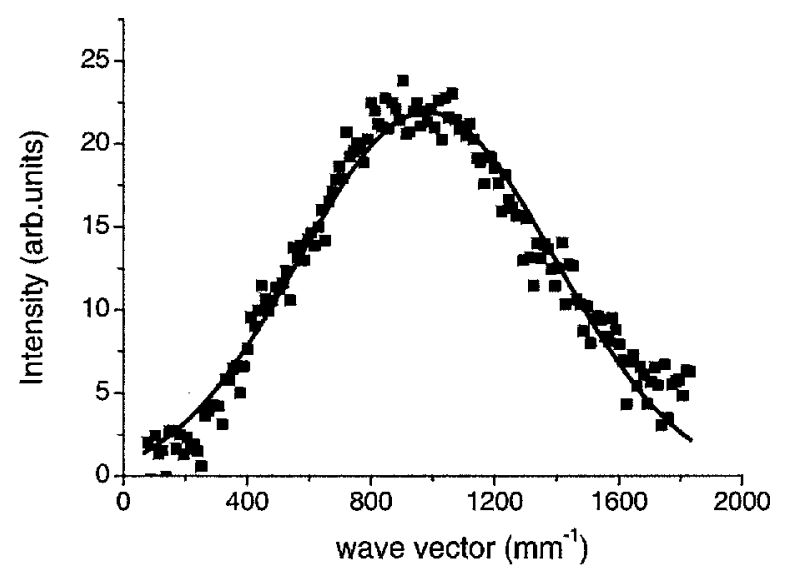

FIG. 4. Scattering pattern of a sample from regime $D$, the curve fits well to a Gaussian, indicating a bell-shaped cluster size distribution as implied by DLCA models.

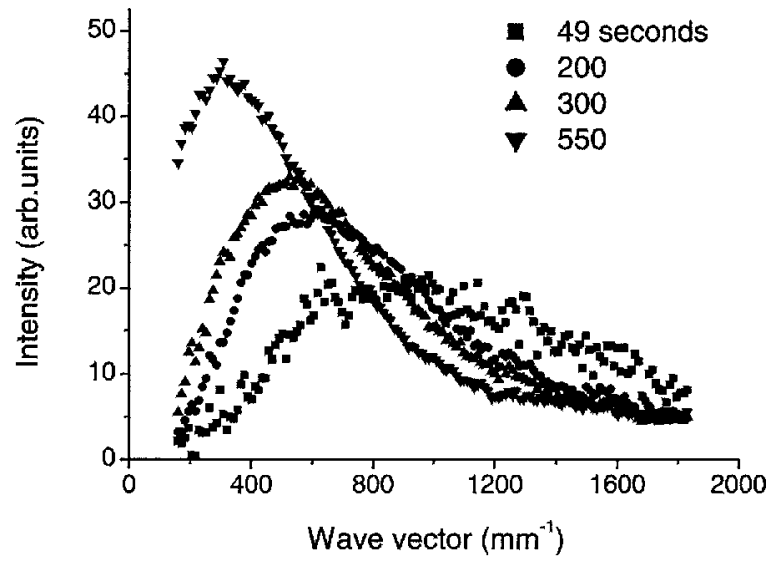

FIG. 5. Scattering pattern of a sample from regime $C\left(c_{p}\right.$ $=1.85 \mathrm{mg} / \mathrm{ml}$ ), the curve is quite asymmetric, this is believed to be due to the polydispersity of size of the clusters.

that smaller particles are indeed still present, in agreement with the RLCA model and previous microscopy work.

In recent years several groups have examined DLCA and RLCA mechanisms using light scattering $[13,20,21]$. One phenomenon that was described was the peak with a sharp decrease in scattering at low $q$. This was ascribed to there being a region around the growing clusters that is depleted of particles, that is, over the volume of the cluster plus depletion region the mass is conserved. The phrase "depletion region" is usually associated with a picture of region of high density, surrounded by a depleted region then a region in which the density returns to the "original" or "normal" value. This picture is not necessarily the case (and in fact for a DLCA mechanism we would not expect it to be so). With a high drive to aggregate it is difficult to imagine how a cluster growing in a sea of other particles would come about. Intuitively we might expect single particles forming doublets, doublets forming quadruplets and so on. The depletion region (that region over which the mass is conserved) then would correspond to the distance midway between clusters as there is no correlation on length scales larger than this and thus no scattering. This combined with the fractal nature of the clusters means it is not obvious to what the peak in the scattering pattern physically corresponds. Figure 2(a) shows a confocal microscopy image typical of a sample undergoing DLCA. It is obvious that there is no depletion region in the sense of there being a region, where the density returns to the original. Some authors ascribe the lengthscale $r$ represented by the peak to the radius of a cluster plus its depletion layer; others interpret the peak as corresponding to the radius of the cluster and the length scale, where the scattering intensity has decreased to the depletion layer. In spite of this ambiguity the variation of the length scale corresponding to the peak position with time is expected to follow a power law ( $r$ $\sim t^{1 / d f}$, where $d_{f}$ is the fractal dimension of the clusters, expected to be 1.8 for DLCA) in the DLCA case and show an exponential growth in the RLCA case $(r \sim \exp (\alpha t)$, where $\alpha$ is a constant) [10]. Similar trends are also found here, as can be seen in Figs. 6 and 7. Figure 6 shows the variation of $r$ for regime $D$, this does show a power law, constraining the power to be 1.8 as predicted then a fit of the form $r=a$ 


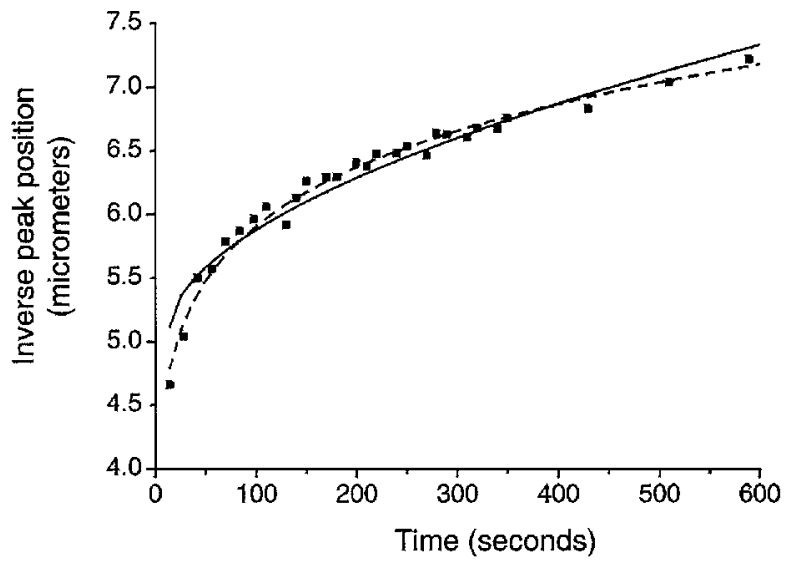

FIG. 6. Time evolution (squares) of the length scales in the diffusion-limited regime $(D)$. The DLCA model predicts a power law growth. Constraining $d_{f}=1.8$ then a fit of $r=a+b\left(t-t_{0}\right)^{1 / d f}$ (solid line) matches the data well. A fit of the form $r=a t^{1 / \gamma}$ with $\gamma=9$ also fits well (dashed line).

$+b\left(t-t_{0}\right)^{1 / d f}$ (where $a, b$, and $t_{0}$ are constants) gives a relatively good fit with $r=\left[5+0.06(t-15)^{1 / 1.8}\right] \mu \mathrm{m}$ this form of the equation would indicate that the mechanism controlling this power law is only valid after a certain time, in this case $t_{0}=15 \mathrm{~s}$. This may be for several reasons, for example, the samples are mixed vigorously to homogenize them before the experiment is started, when the mixing has stopped it must take a finite amount of time for the flows within the sample to die away and slower flows in the sample may then assist aggregation (because the number of particle encounters is increased) thus leading to a delay before the system reaches the conditions that are controlled by a power law. We note, however, that the form $r=b t^{1 / \gamma}$ (where $\gamma$ is a constant, equated above with $d_{f}$ ) gives an excellent fit with $b=3.6$ and $\gamma=9$, though this value of $\gamma$ is obviously far higher than expected. Figure 7 shows the data from a sample in regime

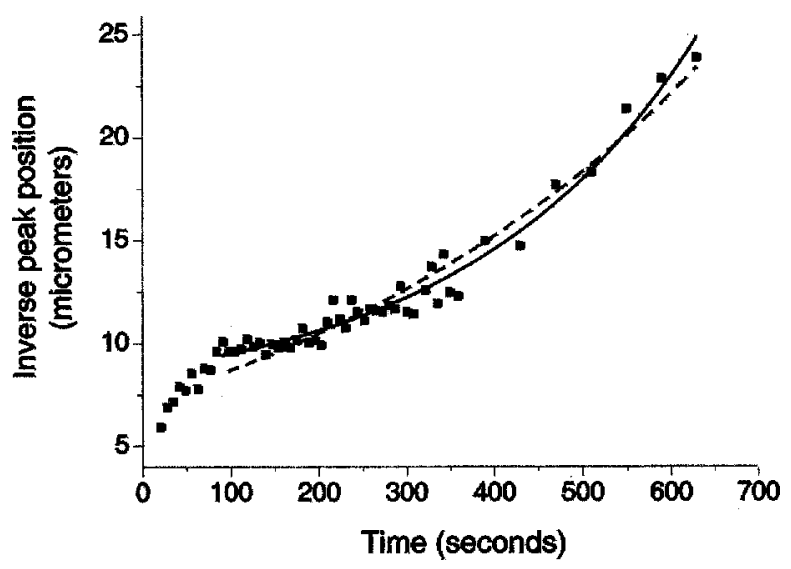

FIG. 7. Time evolution (squares) of the length scales in the reaction-limited regime $(C)$. The RLCA model predicts an exponential growth although this is not seen until after approximately $100 \mathrm{~s}$. A good fit is obtained by allowing for a delay period before exponential growth is seen, $r=e+c \exp \left[d\left(t-t_{0}\right)\right]$ (solid line). The data (after $t=100 \mathrm{~s}$ ) is also fitted to an exponential of the form used in Ref. [10], $r=c \exp (d t)$ (dashed line) but the fit is not as good.
$C$. The data appears to show exponential growth only after an initial period, for which $r$ is almost linear in time. Again including a delay before the growth becomes evident, that is, $r=e+c \exp \left[d\left(t-t_{0}\right)\right]$ (where $c, d$, and $e$ are constants) we get an excellent fit with $r=[7+3 \exp 0.004(t-100)] \mu \mathrm{m}$. If the delay time is not included [that is, if we use the form $r$ $=c \exp (d t)]$ then the fit is not very satisfactory. We have included the details of the fits as it is important to determine which form should be used in fitting the data and it is not always clear in other papers which form is used [for example, Weitz et al. [10] specify using the form $r=c \exp (d t)$ to fit an RLCA regime but only the relation $r \sim t^{1 / d f}$ for the DLCA regime]. Obviously, it is important to determine whether or not there is a delay before these relationships are valid (either because of the mixing, as suggested above, or for other reasons) since this will reveal more details of the very initial stages in the aggregation mechanism.

Figures 3 and 5 (see also Figs. 6 and 7) show a marked difference not only in the shape of the peak but also in the movement of the peak position, the peak moves to much smaller wave vectors in the RLCA regime than in the DLCA regime. This agrees with the findings in Ref. [8], where the structures in regime $D$ were elongated and stringy, those in regime $C$ were much more compact, thus having larger cluster sizes and correspondingly large distances between clusters, this can be seen clearly in Fig. 2.

\section{B. Regime $B$}

In order to look for a nucleation and growth regime, samples with polymer concentrations of $1.81 \mathrm{mg} / \mathrm{ml}$ (just inside regime $C$ ) and less were used.

In a nucleation and growth regime it is expected that there will be a delay time before nuclei are formed and can grow. The scattering at low polymer concentrations was found to be small, it is likely that this is because only a few nuclei were formed, (this proposition is substantiated by the microscopy images shown in the work by de Hoog et al. [8]) and the scattering was not sufficient to make out over the background noise. Thus, we examined the amount of light transmitted (i.e., not scattered) through the sample. Since we use a photodiode to detect the transmitted beam we are, in fact, measuring the power of the remaining unscattered beam $P_{\text {unscat }}$. If there is a delay time there should be a delay in the decrease of $P_{\text {unscat }}\left(P_{\text {unscat }}\right)$ will decrease because of increasing scattering in the sample). We will show here that $P_{\text {unscat }}$ does indeed show a delay time for low polymer concentrations but as the polymer concentration increases the delay time cannot be distinguished above the noise of the data. However, the decrease of $P_{\text {unscat }}$ can be related to the total amount of light scattered, which is in turn related to the growth of the nuclei in the samples. The rate of decrease of $P_{\text {unscat }}$ also varies with the polymer concentration and we attempt to relate this to the distance from the phase boundary.

For polymer concentrations in the range $1.62-1.81 \mathrm{mg} / \mathrm{ml}$ there is no obvious delay time, but the amount of transmitted light appears to decrease immediately. This is shown in Fig. 8. The decrease of $P_{\text {unscat }}$ appears to be linear in time and the 


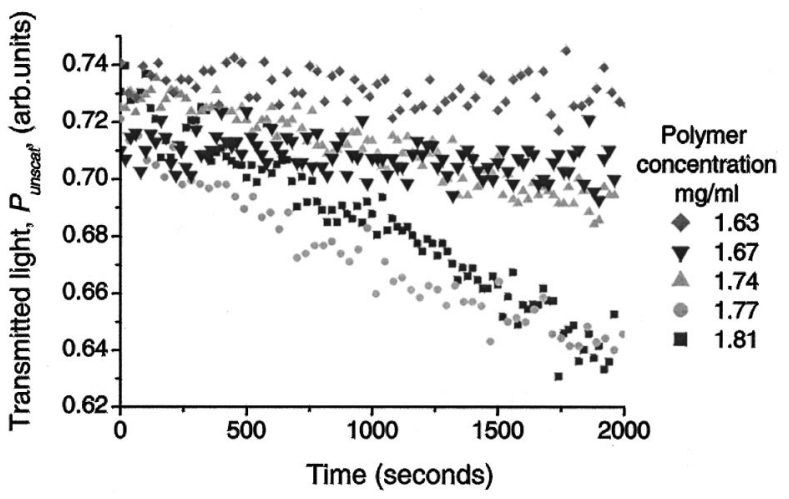

FIG. 8. Change in the power of the unscattered beam (implying an increase in the turbidity of the sample), for samples with low polymer concentration. The decrease is linear with time.

rate of decrease varies with the polymer concentration.

In order to understand the time dependence of $P_{\text {unscat }}$, we need to consider what is happening in the sample: we expect that nuclei are forming and growing larger, thus we examine the time variation of the position wave vector corresponding to the radius of the nuclei $q_{m}(t)$ (we will later relate this to the total amount of light scattered by the sample). We assume here that $q_{m}(t)$ only changes due to cluster growth (that is, cluster nucleation is negligible). We also assume that the probability of a particle attaching itself to the cluster is still small such that any depletion layer around the particle is negligible and we can treat the particle concentration around the cluster as being constant. The rate of decrease of transmitted light is very much smaller than for samples in regime $C$, indicating that the assumption is valid. Thus, we write the change in volume $\left(\sim r^{3}\right)$ in terms of the flux of particles into the cluster (proportional to the surface area $r^{2}$ ) and the probability that the particle remains with the cluster $p$,

$$
\frac{d r^{3}}{d t} \propto p r^{2} \rightarrow r \propto p t \text { thus } q_{m} \propto(p t)^{-1} .
$$

To relate this to the total light scattered, we need to examine the Porod invariant, [22], that is,

$$
\int_{0}^{\infty} l(q) q^{2} d q=\text { const. }
$$

This means that as the peak position shifts to smaller wave vectors the corresponding intensity must increase, but it must increase faster than the wave vector decreases because of the squared term. We will show below that this increase in intensity as the peak moves to smaller wave vectors means that the total amount of light scattered increases. Since we expect the shape of the $I(q, t)$ distribution to be dependent only on a characteristic wave vector $\langle q\rangle$, we can replace the integral by an average intensity $\langle I\rangle$ dependent on the peak position. Thus, we expect $\langle l\rangle=$ const $/\langle q\rangle^{2}$.

The total scattered light, that is, the total power of the light scattered $P_{\text {scat }}$ is given by the integral of the intensity (power per unit area) over the area $S$ over which light is scattered. The geometry is shown in Fig. 9. We need to inte-

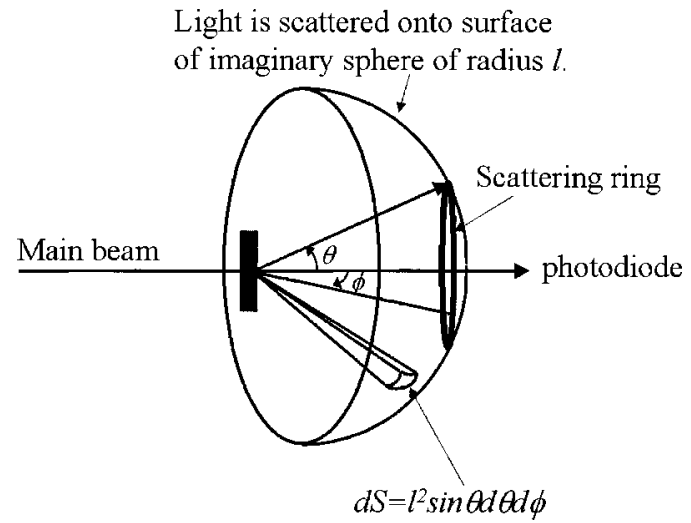

FIG. 9. Scattering geometry used to calculate the total power scattered $P_{\text {scat }}$ and the total power detected by the photodiode $P_{\text {unscat }}$.

grate the intensity over the whole sphere; however, we assume that only small angles are involved (thus, $\sin \theta \sim \theta, q$ $=$ const $\theta / 2$, and so on) and that the scattering is circularly symmetric (thus, terms in $\phi$ and $l$ lead to constants), thus we can write the integral over the area as an integral over $q$

$$
P_{\text {scat }}=\int_{S} I d S \propto \iint_{S} I(\theta, \phi) l^{2} \sin \theta d \theta d \phi \propto \int I(q) q d q .
$$

Again replacing $I(q)$ by a characteristic $\langle I\rangle$, we get the relationship

$$
P_{\text {scat }} \propto 1 /\langle q\rangle .
$$

Equating $\langle q\rangle$ and $q_{m}$ leads to

$$
P_{\text {unscat }}=P_{\text {total }}-P_{\text {scat }}=P_{\text {total }}-A^{\prime} q^{-1}(t)=l_{0}-A p t,
$$

where $A^{\prime}$ and $A$ are constants of proportionality. This predicts a linear time dependence, as found experimentally, the

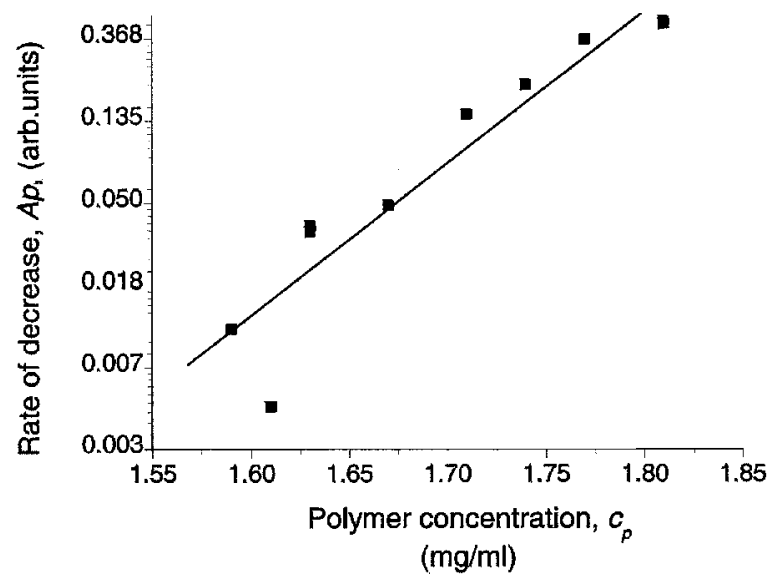

FIG. 10. On decreasing the polymer concentration, the rate of decrease $A p$ of the intensity of the unscattered beam slows. This is as expected, since the drive to phase separate is smaller. The line shows the best fit (of the form $A p=M \exp \left[\left(\alpha c_{p}+\beta\right) / k T\right]$ ) to the data. 


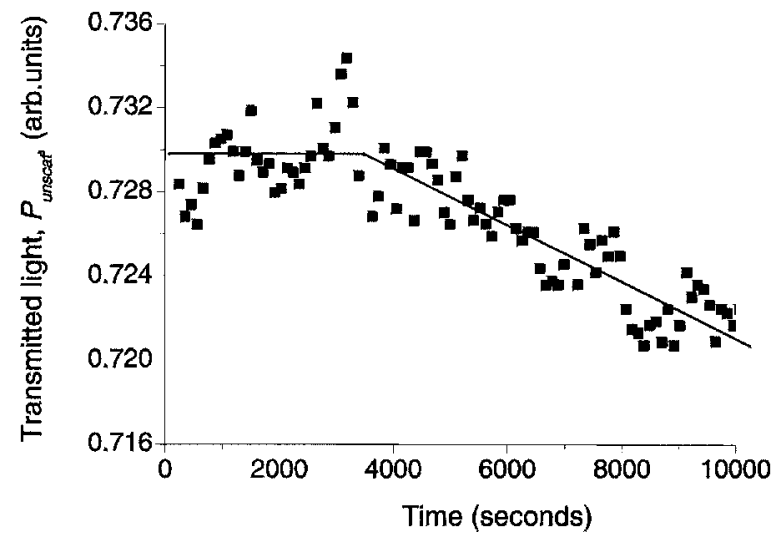

FIG. 11. Scattered intensity of a sample from regime $B$, polymer concentration of $1.59 \mathrm{mg} / \mathrm{ml}$. The intensity of the unscattered beam as a function of time shows a delay before a decrease is seen, as would be expected for a nucleation and growth mechanism.

rate of decrease with time is dependent on $p$ that we would expect to vary as the phase boundary is approached. The rate of decrease $A p$ was measured and plotted as a function of polymer concentration, this is shown in Fig. 10. Indeed the rate does become smaller on approach of the phase boundary (from the previous work this is believed to be at $c_{p}^{*}$ $=1.58 \mathrm{mg} / \mathrm{ml}$ ). Writing $\Delta$ as the energy difference on adding a particle to the cluster, then we expect that $p \sim \exp$ $(-\Delta / k T)$. We expect that $\Delta$ will increase as the polymer concentration increases, the simplest form is then $\Delta=\alpha c_{p}+\beta$, where $\alpha$ and $\beta$ are constants. Thus,

$$
p \propto \exp \left(\frac{\alpha c_{p}+\beta}{k T}\right) .
$$

The measured rate $A p$ as a function of $c_{p}$ is shown in Fig. 10 and the predicted variation agrees with the data very well.

Only for a polymer concentration of $1.59 \mathrm{mg} / \mathrm{ml}$ was a delay time observed, this is shown in Fig. 11. Figure 12 (showing the data for a sample of $c_{p}=1.71 \mathrm{mg} / \mathrm{ml}$ ) also suggests that a delay is present but it is difficult to quantify it reliably. At polymer concentrations just inside regime $B$ the decrease in $P_{\text {unscat }}$ is very slow and the data is very noisy, thus a delay time may be present for polymer concentrations higher than $1.59 \mathrm{mg} / \mathrm{ml}$ but cannot be measured with the equipment.

\section{CONCLUSIONS}

The light scattering data has confirmed the regimes classified by de Hoog et al. [8]. The techniques of microscopy

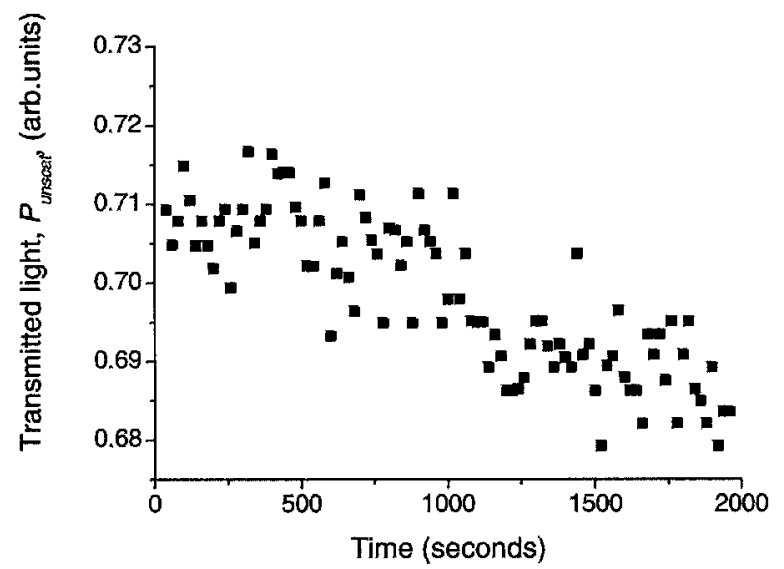

FIG. 12. Change in intensity of an unscattered beam for a sample in regime $B\left(c_{p}=1.71 \mathrm{mg} / \mathrm{ml}\right)$. The difficulty, due to noise, in determining the presence or absence of a delay time can be seen. Certainly the data does not preclude the existence of delay time.

and light scattering provide important complementary data. The former allows analysis in terms of Schmolukowski kinetics, as well as a direct insight into the local aggregate structure, permitting phenomena such as structure rearrangement (a significant possibility though often assumed negligible in simulation) to be detected. The light scattering allows for a more quantitative characterization and can be compared more easily with the predictions of earlier works and gives better statistics on the three-dimensional structure. We have compared our results with those predicted for DLCA, RLCA, and nucleation and growth regimes and have shown conclusively that these regimes are present. The growth in regime $B$ has been analyzed in terms of the reaction probability using an energy dependent on the polymer concentration. In addition to confirming the regimes expected several points have arisen that indicate directions for future research. The first is the variation of the length scale $r$ corresponding to the peak position. The physical relationship between $r$ and the depletion and core regions needs to be examined further, in addition, investigation of the time variation of $r$ in the different regimes and possibility of a delay before a specific variation is seen is likely to provide valuable information on the scope of the validity of different mechanisms.

\section{ACKNOWLEDGMENTS}

The authors would like to thank Carel van der Werf, who has been a vital source of assistance with the small angle light scattering apparatus and software. We also appreciate valuable discussions regarding the transmission data with Andrei Petukhov and his critical reading of the paper.
[1] S. Asakura and F. Oosawa, J. Chem. Phys. 22, 1255 (1954).

[2] A. Vrij, Pure Appl. Chem. 48, 471 (1976).

[3] N. A. M. Verhaegh and H. N. W. Lekkerkerker, in The Physics of Complex Systems, Proceedings of International School of Physics "Enrico Fermi," Course CXXXIV, edited by F. Mal- lamace and H. E. Stanley (IOS, Amsterdam, 1997), p. 347.

[4] W. v. Megen and S. M. Underwood, Phys. Rev. E 49, 4206 (1994).

[5] N. A. M. Verhaegh, D. Asnaghi, and H. N. W. Lekkerkerker, Physica A 264, 64 (1999). 
[6] C. Smits, B. v. d. Most, J. K. G. Dhont, and H. N. W. Lekkerkerker, Adv. Polym. Sci. 42, 33 (1992).

[7] W. C. K. Poon and M. D. Haw, Adv. Colloid Interface Sci. 73, 71 (1997).

[8] E. H. A. de. Hoog, W. K. Kegel, A. v. Blaaderen, and H. N. W. Lekkerkerker, Phys. Rev. E (to be published).

[9] P. Meakin, in Phase Transitions and Critical Phenomena, edited by C. Domb and J. L. Lebowitz (Academic, New York, 1988), Vol. 12, p. 336.

[10] D. A. Weitz, J. S. Huang, M. Y. Lin, and J. Sung, Phys. Rev. Lett. 54, 1416 (1985).

[11] M. Y. Lin, H. M. Lindsay, D. A. Weitz, R. C. Ball, R. Klein, and P. Meakin, Phys. Rev. A 41, 2005 (1990).

[12] D. Asnaghi, M. Carpinetti, M. Gigkio, and M. Sozzi, Phys. Rev. A 45, 1018 (1992).

[13] D. Asnaghi, M. Carpineti, and M. Giglio, Mater. Res. Bull. 19, 14 (1994).

[14] M. Lach-hab, A. E. Gonzalez, and E. Blaisten-Barojas, Phys.
Rev. E 54, 5456 (1996).

[15] M. D. Haw, W. C. K. Poon, and P. N. Pusey, Phys. Rev. E 56, 1918 (1997).

[16] G. Feher and Z. Kam, Methods Enzymol. 114, 77 (1985).

[17] L. Antl, J. W. Goodwin, R. D. Hill, R. H. Ottewil, S. M. Owens, and S. Papworth, Colloids Surf. 17, 67 (1986).

[18] G. Bosma, C. Pathmamanoharan, E. H. A. de. Hoog, W. K. Kegel, A. v. Blaaderen, and H. N. W. Lekkerkerker, J. Interface Science (to be published).

[19] K. Schatzel and B. J. Ackerson, Phys. Rev. E 48, 3766 (1993).

[20] A. E. Gonzalez and G. Ramirez-Santiago, Phys. Rev. Lett. 74, 1238 (1995).

[21] W. C. K. Poon, A. D. Pirie, M. D. Haw, and P. N. Pusey, Physica A 235, 110 (1997).

[22] O. Glatter, in Modern Aspects of Small Angle Scattering, edited by H. Brumberger (Kluwer Academic, Dordrecht, The Netherlands, 1995), Vol. 451. 